\title{
Haemorrhagic Gingival Hypertrophy: A Striking Presentation of Acute Myelomonocytic Leukaemia
}

\author{
Hipertrofia Gengival Hemorrágica: Apresentação Impressionante de Leucemia \\ Mielomonocítica Aguda
}

Pedro Eduardo SILVA $\triangle^{1,2}$, Alexandra MONTEIRO ${ }^{3}$, António SANTOS CASTRO ${ }^{1}$

Acta Med Port 2015 Sep-Oct;28(5):675-675

Keywords: Gingival Hypertrophy; Leukemia, Myelomonocytic, Acute.

Palavras-chave: Hipertrofia Gengival; Leucemia, Mielomonocítica, Aguda.

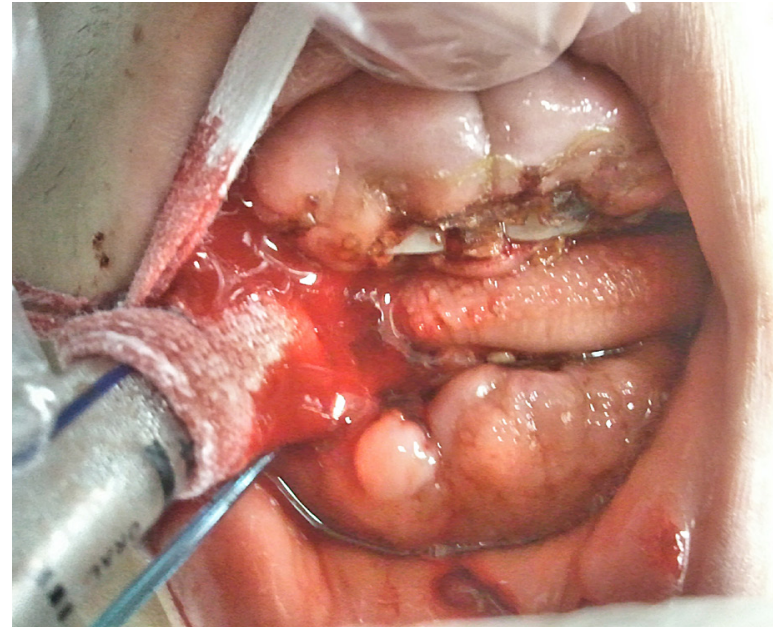

Figure 1 - Haemorrhagic gingival hypertrophy with ulcerated lesions in the labial mucosa

A 19 year-old male with unremarkable previous history presented to the primary care physician with gum pain and swelling five weeks before admission. Antibiotics were started considering a dental infection. Since clinical deterioration ensued, he was referred to our hospital presenting cachexia, a Glasgow Coma Scale of 9, dysarthria, left hemiparesis, jaundice, hepatosplenomegaly, enlarged lymph nodes, oral ulceration, and a classical sign: haemorrhagic gingival hypertrophy (Fig. 1)., Laboratory results: haemoglobin $8.3 \mathrm{~g} / \mathrm{dL}, \mathrm{WBC} 359,100 \times 10^{\wedge} \mathrm{g} / \mathrm{L}(70 \%$ blasts), platelets $60 \times 10^{\wedge} 9 / \mathrm{L}$, INR 10.01, uric acid $6.8 \mathrm{mg} /$

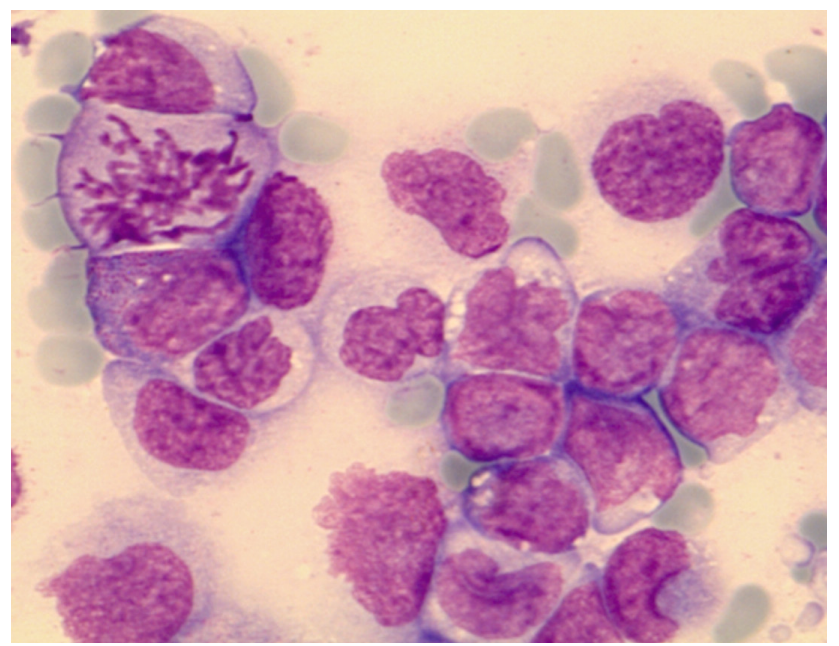

Figure 2 - May-Giemsa stain showing almost exclusively blasts, including one mitosis

$\mathrm{dL}$, total bilirubin $2.34 \mathrm{mg} / \mathrm{dL}$, LDH $4077 \mathrm{U} / \mathrm{L}$. Brain CTscan: right frontal and right cerebellar hematomas.

Bone marrow smear presented $89 \%$ of myeloperoxidase-positive blast cells, consistent with an acute myelomonocytic leukaemia (Fig. 2). Immunophenotypic analysis showed $60 \%$ of monocyte-derived dendritic cells, compatible with an aggressive and rare form of leukemia acute myeloid dendritic cell leukemia. ${ }^{3,4}$

Despite prompt systemic induction chemotherapy with cytarabine, the patient died 4 days after arrival on the intensive care unit.

\section{REFERENCES}

1. Brenneise CV, Mattson JS, Commers JR. Acute myelomonocytic leukemia with oral manifestations: report of case. J Am Dent Assoc. 1988;117:835-7.

2. Hou G, Huang J, Tsai C. Analysis of oral manifestations of leukemia: a retrospective study. Oral Dis. 1997;138:31-8.

3. Lichtman MA, Segel GB. Uncommon phenotypes of acute myelogenous leukemia: Basophilic, mast cell, eosinophilic, and myeloid dendritic cell subtypes: A review. Blood Cells Mol Dis. 2005;35:370-83.

4. Vardiman JW, Thiele J ADEA. The 2008 revision of the WHO classification of myeloid neoplasms and acute leukemia: rationale and important changes. Blood. 2009;114:937-52.

\footnotetext{
1. Department of Internal Medicine 2.3. Hospital de Santo António dos Capuchos. Centro Hospitalar de Lisboa Central. Lisboa. Portugal.

2. CEDOC, Chronic Diseases Research Centre. NOVA Medical School. Faculdade de Ciências Médicas. Universidade NOVA de Lisboa. Lisboa. Portugal.

3. Departament of Haematology. Hospital de Santo António dos Capuchos. Centro Hospitalar de Lisboa Central. Lisboa. Portugal.

$\square$ Autor correspondente: Pedro Eduardo Silva. pedro.eduardo.silva@gmail.com

Recebido: 02 de Março de 2015 - Aceite: 07 de Setembro de 2015 | Copyright $\odot$ Ordem dos Médicos 2015
} 
Pedro Eduardo SILVA, Alexandra MONTEIRO, António SANTOS CASTRO

\section{Haemorrhagic Gingival Hypertrophy: A Striking Presentation of Acute Myelomonocytic Leukaemia}

Acta Med Port 2015:28:675-675

Publicado pela Acta Médica Portuguesa, a Revista Científica da Ordem dos Médicos

Av. Almirante Gago Coutinho, 151

1749-084 Lisboa, Portugal.

Tel: +351218428 215

E-mail: submissao@actamedicaportuguesa.com

www.actamedicaportuguesa.com

ISSN:0870-399X | e-ISSN: 1646-0758

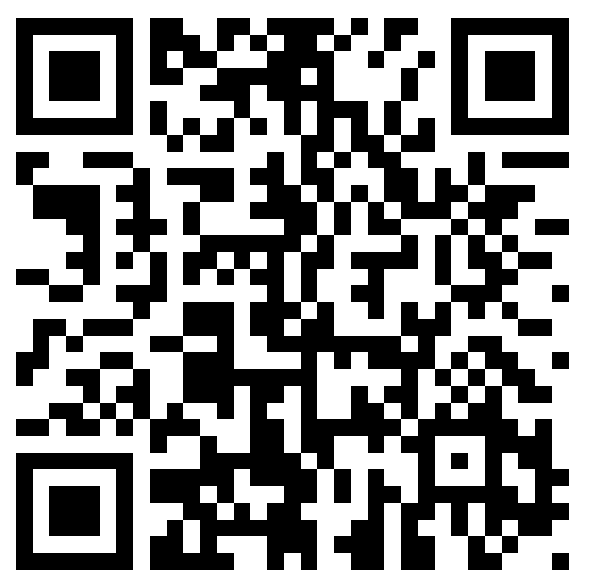

